\title{
SENSOR TECHNOLOGY FOR INTEGRATED VEHICLE HEALTH MANAGEMENT OF AEROSPACE VEHICLES
}

\author{
W. H. Prosser, T. L. Brown, S. E. Woodard, G. A. Fleming, and E. G. Cooper \\ NASA Langley Research Center, Hampton, VA 23681
}

\begin{abstract}
NASA is focusing considerable efforts on technology development for Integrated Vehicle Health Management systems. The research in this area is targeted toward increasing aerospace vehicle safety and reliability, while reducing vehicle operating and maintenance costs. Onboard, real-time sensing technologies that can provide detailed information on structural integrity are central to such a health management system. This paper describes a number of sensor technologies currently under development for integrated vehicle health management. The capabilities, current limitations, and future research needs of these technologies are addressed.
\end{abstract}

\section{INTRODUCTION}

The application of traditional NDE methods for on-ground inspection of aerospace vehicles contributes greatly to their safety and reliability. However, periodic inspections significantly increase operating expense and vehicle processing time. Further, the need to disassemble and reassemble structural components to allow inspections can lead to damage or degradation of the structure or auxiliary systems (e.g., electrical wiring and hydraulic lines). NASA is focusing on technology development for Integrated Vehicle Health Management (IVHM) systems to address these issues, and to meet demanding goals in increasing aerospace vehicle safety and reliability while reducing vehicle operating costs. On-board, real-time sensing systems for structural integrity assessment are central to the IVHM approach. Such sensing systems will minimize the need for periodic NDE inspections, or at least focus these inspections to specific vehicle areas where damage was indicated. Sensors comprising an IVHM system must be able to withstand harsh aerospace operating environments, while having minimal size, weight, and power requirements. Several candidate sensor technologies for use in an IVHM system are discussed in this paper. These include fiber-optic sensors, active and passive ultrasonic methods, remote wireless technologies, and remote non-contact sensing. Additionally, a brief discussion on IVHM system architecture is provided to illustrate the considerations given to establishing architectures capable of handing the data acquisition, processing, analysis, and storage for massive numbers of multiple sensor types.

\section{FIBER OPTIC SENSORS}

Considering the large acreage of aerospace vehicle structural elements, it is a given that extremely large numbers of sensors will be required for on-board structural integrity assessment. Fiber optic sensors have been identified as the leading candidate technology 
for meeting this requirement with minimal weight penalty. Numerous sensor sites can be multiplexed along a single optical fiber, mitigating the complexity and weight inherent with the wiring required for a large number of single ended sensors. Fiber optic sensors also provide other advantages such as the ability to measure many different structural parameters of interest, immunity to electromagnetic interference (EMI), and the ability to operate over very large temperature environments.

Fiber optic sensors can be separated into two classes for discrete strain and temperature measurement: cavity-based designs and grating-based designs [1]. Cavitybased designs utilize an interferometric cavity in the fiber to create the sensor. Examples include the extrinsic Fabry-Perot interferometer (EFPI), the intrinsic or fiber Fabry-Perot interferometer (IFPI or FFPI), and all other etalon-type devices. Although such sensor designs have been utilized in a wide variety of applications such as in high temperature and EMI environments, they do not allow for multiplexing capability in a single fiber, and thus may be limited for applications requiring large number of sensors.

Grating-based designs utilize a photo- or heat-induced periodicity in the fiber core refractive index to create a sensor whose reflected or transmitted wavelength is a function of this periodicity. Grating-based sensors (e.g., Bragg gratings) can be easily multiplexed by using gratings of different wavelength as in the case of wavelength division multiplexing (WDM). Factors limiting the number of sensors in a single fiber include the limited bandwidth of the source as well as that supported by the fiber, and the range over which the physical parameter of interest is being measured.

Another grating-based system developed at NASA Langley $[2,3]$ has the ability to multiplex hundreds or thousands of Bragg gratings (with the same wavelength) in a single fiber. The system is based on the principle of optical frequency domain reflectometry (OFDR) and essentially eliminates the bandwidth limitations imposed by the WDM technique. Figure 1 shows results obtained when the system was used to measure strains in composite coupons subjected to combined thermal (elevated and cryogenic) and mechanical loading. The plots compare measurements obtained using the fiber optic sensors versus traditional resistance strain gages. Three optical fibers, each containing Bragg gratings, were bonded to the surface adjacent to two conventional foil strain gages. A single resistive thermal measurement device was in close proximity to the strain gages.

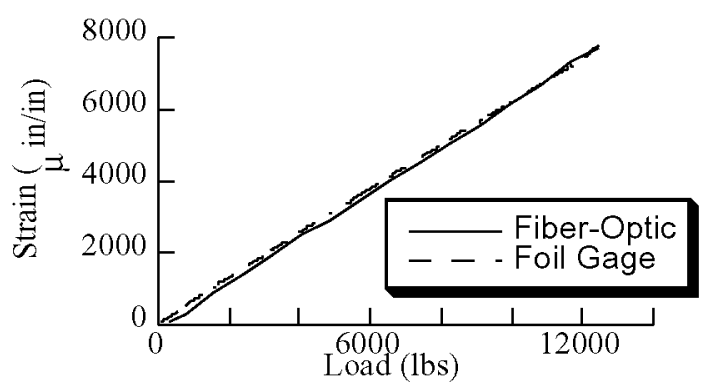

a) IM7/977-2, Tensile, $-320^{\circ} \mathrm{F}$.

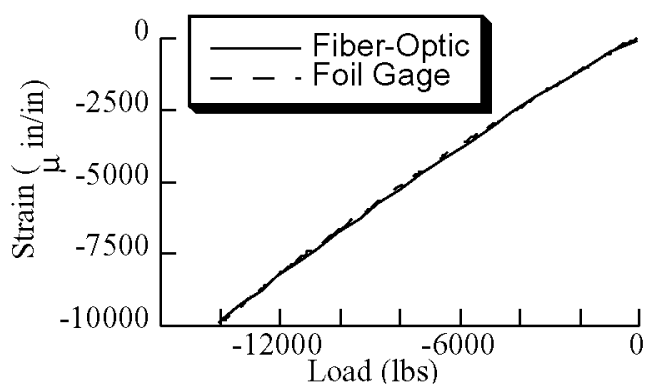

b) IM7/977-2, Compressive, $-320^{\circ} \mathrm{F}$.

Figure 1. Comparison of strain measurements from Bragg gratings and foil strain gages.

The graphs in Figure 1 contain tension/compression data for IM7/977-2 composite specimens subjected to $-320^{\circ} \mathrm{F}$ temperatures. The dashed line trace in each of the graphs represents the data from the fiber Bragg gratings while the solid trace represents data from the conventional foil strain gages. The horizontal axis displays load values in poundsforce while the vertical axis displays strain in microinches per inch. The fiber optic sensors show excellent agreement with the foil gages. At elevated temperatures $\left(500^{\circ} \mathrm{F}\right)$, 
in tests of T650-35/PMR-15 composite specimens, a similarly good agreement between foil gage and fiber optic sensors was seen in compressive loading. Some small discrepancies were observed for tensile testing, possibly due to fiber-optic thermal compensation effects, and are under further investigation.

The preliminary findings from these thermo-mechanical tests indicate that fiber Bragg gratings are capable of accurately measuring tensile and compressive strain at elevated and cryogenic temperatures, although compensation for the effect of temperature on the optical parameters of the Bragg gratings may be necessary, particularly at elevated temperatures. Additional research areas that are of concern include: 1) adhesive selection and bonding procedures for surface mounting the fiber optic sensors, 2) embedded fiber optic sensor characterization at elevated and cryogenic temperatures, and 3) transverse sensitivity of fiber optic sensors. These areas will continue to be explored in future research to support aerospace vehicle requirements.

\section{ACTIVE AND PASSIVE ULTRASONIC SENSING}

Ultrasonic sensing, applied in both active and passive modes, is another sensor technology area receiving considerable attention. Analysis of actively transmitted ultrasonic signals is a conventional NDE methodology that has long been used to detect and assess damage. However, such approaches use sensors that are scanned over the structure to provide a point-by-point representation of material properties and/or damage locations. Such scanning probe approaches are not currently feasible for continuous, onboard monitoring. Therefore, the use of arrays of permanently attached or embedded ultrasonic transducers, which act dually as transmitters and receivers, is being researched. Ultrasonic signals generated by one transducer are detected by neighboring transducers within an array. Damage along paths between the transducers can be detected, and with more complex analysis methods, material along secondary propagation paths that include reflections from structural boundaries can also be evaluated. The development of the Stanford Multi-Actuator Receiver Transduction (SMART) layer is an excellent example of recent efforts in this area [4]. Ongoing areas of research in active ultrasonic sensing technology for structural health monitoring include 1) the further improvement and characterization of miniaturized, rugged, embeddable sensors, 2) analysis methodologies for optimized sensor placement to enable characterization of damage throughout the entire structure rather than just along direct propagation paths, and 3) modeling of ultrasonic guided wave propagation that occurs when such sensors are attached or embedded on thinwalled aerospace structures.

Passive ultrasonic monitoring, also known as acoustic emission (AE), also utilizes an array of ultrasonic sensors. The sensor array is used to passively monitor acoustic signals generated by damage mechanisms such as crack growth. AE is widely used as a conventional method for off-line structural assessment, and can also be implemented insitu to monitor a structure while in service. This capability makes it well suited for onboard structural health monitoring of aerospace vehicles. However, considerably more research and development is required to make $\mathrm{AE}$ a more viable technology for IVHM. Successful implementation of AE requires sensors having lighter weight, increased sensitivity, and increased ruggedness over those currently available. Additionally, reductions in size, weight, and power requirements of the associated AE monitoring instrumentation are also needed. Advances in AE analysis methodologies are required to more accurately locate and identify damage, while intelligently discriminating extraneous noise from signals indicating actual damage. Ongoing efforts in this field include the development of $\mathrm{AE}$ multiplexing instrumentation that can miniaturize $\mathrm{AE}$ flight systems, 
the development of fiber optic AE sensors [5] and the development of Modal AE based analysis methods [6]. Another significant development is that of modeling approaches to better understand and predict AE propagation phenomena [7]. Such models are of benefit for a number of reasons to include the characterization of AE transducers, optimization of sensor placement on a structure, scaling of $\mathrm{AE}$ results from laboratory test coupons to full scale structures, and the development of new and automated AE data analysis methods.

\section{WIRELESS REMOTE SENSOR SYSTEMS}

Conventional sensors such as strain gages, thermocouples, and accelerometers will also be used for structural health monitoring. One major issue for such sensors is the need to route large numbers of wires to provide power and data communication. This is an especially difficult problem when retrofitting these sensors into existing structures, such as the aging aircraft fleet. To address this concern, a prototype adaptable vehicle healthmonitoring architecture has been developed [8] and flight tested. The architecture is selfcontained and requires limited integration intrusion into existing systems, having "bolton/bolt-off" simplicity. There are three operational levels to the architecture: one or more remote data acquisition units (RDAU) located throughout the vehicle; a command and control unit (CCU) located within the vehicle; and, a terminal collection unit (TCU) to collect analysis results from all vehicles.

The RDAUs are multi-sensor interfaces with an on-board miniature computer, programmable digital interface, nonvolatile solid-state memory and a wireless transceiver for communication with the command and control unit. Communication is achieved by using wireless radio frequency transceivers operating at $433 \mathrm{MHz}$. The RDAUs were designed to withstand impact during aircraft landing while mounted on the main landing gear, and have been vibration tested up to an acceleration amplitude of $20 \mathrm{~g}$ at $2000 \mathrm{~Hz}$. It was also designed to operate in non-environmentally controlled locations of the plane. The RDAU was thermally tested for temperatures ranging from $-50^{\circ} \mathrm{C}$ to $55^{\circ} \mathrm{C}$ and

pressure tested to simulate $50,000 \mathrm{ft}$ altitude. Vibration tests verified that the remote data acquisition unit could operate at vibration levels representative of those experienced by commercial aircraft. During vibration testing, the final acceleration amplitude was $20 \mathrm{~g}$ at $2000 \mathrm{~Hz}$. The remote data acquisition unit has an eight channel programmable digital interface, which allows the user discretion in choosing type of sensors, number of sensors, sensor sampling rate and sampling duration for each sensor. Programmable data acquisition circuitry and expert systems trained to performance baselines in each RDAU allow the architecture to be adaptable for many types of vehicles and structures. Once a suite of sensors has been chosen for each RDAU and installed on the vehicle, a baseline of acceptable vehicle performance is established from measurements acquired when the vehicle is performing correctly. Each RDAU uses an embedded expert system trained to its respective baseline

The CCU is a computer-based subsystem that provides the communications, analysis repository, and user interface functions for the RDAUs. The CCU can also serve as a power management tool by regulating when individual or combinations of RDAUs are powered. A simple radio frequency (RF) wireless network of RDAUs can be controlled from a single CCU. The TCU provides the means to autonomously retrieve vehicle analysis results from all vehicle CCUs. The TCU performs analysis on results collected from all vehicles to identify any fleet-wide anomalies (e.g., all aircraft have the same faulty bearing at a similar location). The TCU develops the final summary of the vehicle health monitoring results that gets routed to the appropriate users (e.g., maintenance workers, airlines operations, etc.). 
This architecture system has been flight tested on NASA Langley's Airborne Research Integrated Experiments System (ARIES). There were 13 flight tests of the RDAU and CCU. The flight tests were performed to validate the following: the wireless radio frequency communication capabilities of the system, the hardware design, command and control, software operation, and, data acquisition, storage, and retrieval. A very rigorous test of the mechanical design was achieved by mounting the device on the left main landing gear. During the initial flight tests, none of the autonomous features had been installed. The system functioned as a remotely controlled data acquisition device. Measurements acquired during flights included take-offs, landings, vibration while gear was fully retracted, taxiing, and, touch and go landings. The flight tests demonstrated that the remotely controlled data acquisition capability worked correctly.

\section{NON-CONTACT SENSOR SYSTEMS}

Although most current visions of structural health monitoring systems are based on sensors that are attached to or embedded within the structure, the adaptation of noncontacting measurement systems should not be ruled out. Methods such as laser vibrometry [9], shearography [10], laser ultrasound [11], and infrared thermography [12] are examples of these techniques. These methods are typically applied externally to a structure to interrogate specific vehicle components where damage may have occurred. As such, they satisfy a critical role as part of an integrated vehicle health management system by providing enhanced ground-based diagnostic capabilities. These techniques can thus be used to validate fault indicators or damage sites identified by the on-board sensor systems. Further, there is potential that in the future such non-contact sensor systems could be incorporated into some aerospace structural systems, such as large space platforms.

The use of structural vibration signatures as an indicator for airframe integrity is a growing field [13]. Vibration signatures are typically acquired at a single or small set of points on the aircraft surface using either a scanning laser vibrometer or accelerometers. The surface vibration data are acquired in response to an impulse force or frequency chirp applied by an excitation source at several locations about the vehicle. Comparison of the time-frequency and/or wavelet analyses of the signals obtained in baseline and aged conditions can lead to the identification of airframe cracks, disbonds, or fatigue [14-15].

Measuring the vibration signatures at only a few select points can often cause difficulties in determining the locus of damage. Therefore, it is desirable to acquire measurements at multiple points simultaneously. This enables spatial-temporal cross correlations between the data obtained at each measurement site, yielding improved accuracy in determining the location of airframe flaws. These capabilities are currently being pursued by the development of a multi-point laser vibrometer.

Figure 2 shows the multi-point laser vibrometer configuration currently being pursued for the acquisition of vibration signatures over a two-dimensional array of measurement sites. Contrary to conventional scanning laser Doppler vibrometry (SLDV), the laser beam is not scanned from measurement site to measurement site. The timedependent surface vibration is measured at each measurement site simultaneously so that vibration transients are preserved. Measurement site locations are generated by passing the output laser beam through a diffractive optical element, which can be fabricated to split the beam into any desired pattern with better than $90 \%$ efficiency and uniformity. Doppler-shifted scattered light is collected from each measurement location on the vibrating surface using a standard video camera lens, and mixed with reference light derived from the fundamental laser beam. The resulting light energy is intensitymodulated at the Doppler shift frequency experienced at each measurement location. The 
intensity-modulated light is subsequently focused to discrete sensor locations and digitized to obtain the time-dependent vibration signature. The data are further processed off-line to examine spatial-temporal cross correlation patterns for NDE purposes.

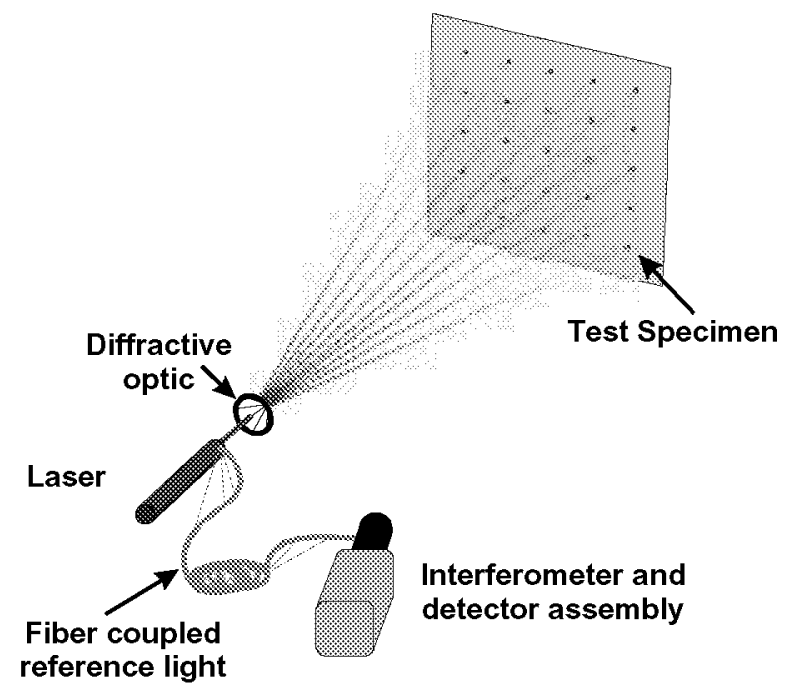

Figure 2. Schematic of multi-point laser vibrometer for simultaneous measurements over a two-dimensional area

Figure 3 shows a prototype multi-point vibrometer designed to measure the propagation of structural vibrations along a line. The object under test was a 4.9-meter long, 1.2-meter diameter aluminum cylinder fabricated in the same manner as an aircraft fuselage. Surface vibration measurements in response to an applied impulse force were obtained at 512 individual sensor sites along the 0.4-meter interrogation line. An example plot showing the time-dependent surface wave propagation is shown in figure 4.

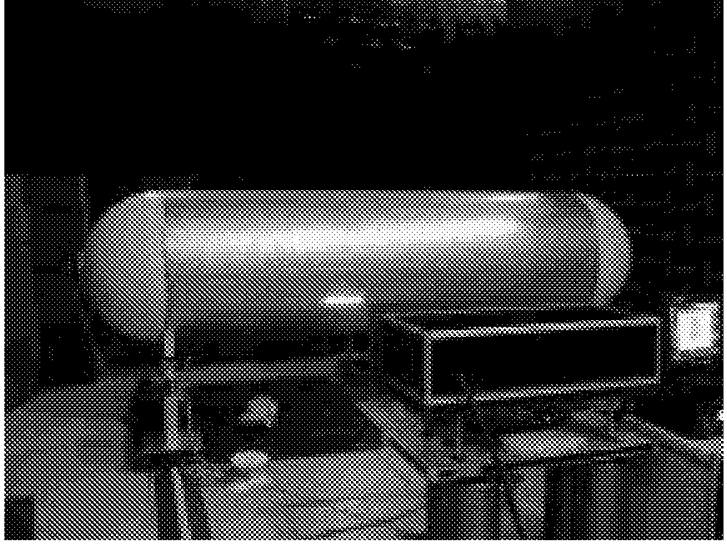

Figure 3. A 1-dimensional multi-point vibrometer

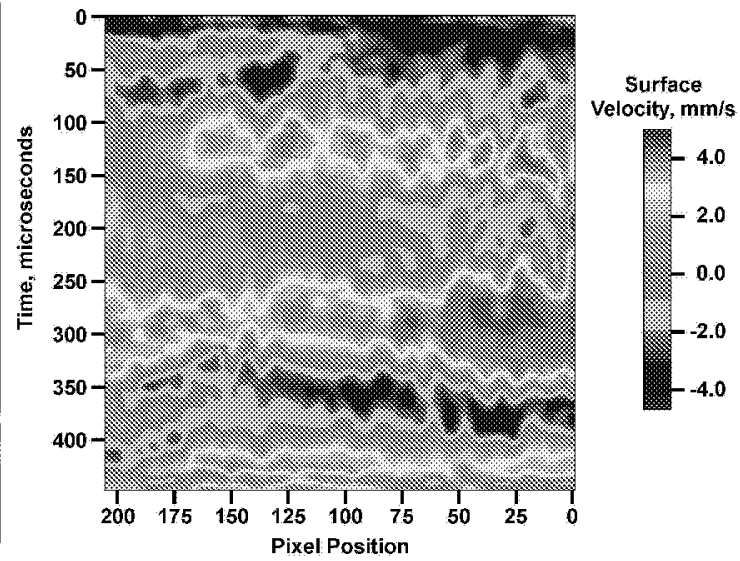

Figure 4. Vibration measurements across the interrogation line after impulse force

\section{ARCHITECTURE CONSIDERATIONS}

In addition to considering the types of sensors required to characterize structural integrity as part of an IVHM system, the data systems and processing architectures necessary to support such large numbers of heterogeneous sensors must also be considered. The architecture will be highly complex, as it must provide for the 
interrogation, digitizing, pre-processing, and archiving of massive amounts of raw signal information for consumption by modeling and analysis modules that will assess the integrity of the affected structural elements. Furthermore, since it is anticipated that a portion of the cost benefits gained through the deployment of on-board SHM systems is achieved through the elimination of certain maintenance and inspection procedures, the architecture's level of reliability must be commensurate with current regulatory guidance for assuring continued airworthiness $[16,17]$. The magnitude of raw signal data, coupled with the complexity of the network interconnections and evolving diagnostic and prognostics methodologies, necessitate key architecture characteristics of scalability, robustness, flexibility, and maintainability [18].

Recognizing that architecture cannot be completely separated from application, work is underway to define a methodology that will aid in designing architectures for IVHM environments, and a layered reference architecture that facilitates scalability, robustness, flexibility, and maintainability $[18,19]$ is being developed. It is anticipated that a SHM architecture will support a data flow that includes real-time flight data (e.g., altitude, airspeed, accelerations, etc.) and sensor data (e.g., acoustic emission, strain, vibration, corrosion, etc.) that is tagged and conditioned (e.g., when, where, amount, rate, etc.), archived for trend analysis and usage history, then forwarded to a flight profiler for determination of phase-of-flight and maneuver. The tagged and conditioned data, coupled with flight profile, usage history, certification load data, and archived maintenance data, is then made available to the diagnostics/prognostics modules for degradation assessment.

NASA is currently giving specific emphasis to architectures supporting the deployment of Langley's OFDR fiber optic Bragg grating sensor system technology as a key sensor suite component for on-board structural health and usage monitoring. A series of simulated axial fuselage lap joints have been instrumented with Bragg gratings and tested at NASA Langley for purposes of developing an architecture concept as well as building a proof-of-concept diagnostic inference model that can infer the presence of growing fatigue cracks at affected and adjacent fasteners [20]. As a result of these preliminary tests, several key architecture areas were identified as needing further investigation including (1) reduction, representation, and archival of large data sets suitable for retrieval by current degradation and damage assessment modules, (2) optimal techniques for increasing timeliness in demodulating the waveform, including dedicated distributed processors and analog techniques, (3) automatic identification and location of Bragg gratings within each fiber string, (4) miniaturization of components for sub-system distribution throughout the airframe, (5) fusion of fiber optic strain sensor data with other pertinent sensor information, and (6) architecture compatibility between laboratory test environments and flight-worthy avionics [21].

\section{SUMMARY}

Extremely large numbers of a variety of sensor types will be necessary to provide real-time, on-board structural integrity assessment as part of an IVHM system for aerospace vehicles. These sensors will measure a multitude of parameters including strain, temperature, load, pressure, vibration, ultrasonic waves, and local chemistry. For flight applications, such sensors will need to be extremely lightweight, as well as be able to survive rugged environments. At present, fiber optic sensing is the leading candidate for such applications because of the ability to multiplex hundreds to thousands of sensors in a single fiber. Ultrasonic sensors, utilized in both active and passive modes, are also being studied for onboard structural health monitoring. For retrofit onto existing vehicles, a remote wireless sensor architecture is being developed that can support a variety of conventional sensor 
types, and be bolted into locations on vehicles without having to route wires to provide communication and power. Remote, non-contacting sensor technologies are being developed for complimentary ground inspections, and possibly for on-vehicle deployment. Further, the data systems and processing architectures that will be required to support these massive numbers of diverse sensors are being considered, with special emphasis on the integration of fiber optic sensors with more conventional sensor types.

\section{REFERENCES}

1. Steenkiste, R. J. and Springer, G. S., Strain and Tempertaure Measurement with Fiber Optic Sensors, Technomic Publishing (Lancaster, PA) 1997, pp. 3-4.

2. Froggatt, M. and Moore, J., in Applied Optics, vol. 37, No. 10, 1998, pp. 1741-1746.

3. Childers, B. A., Froggatt M. E., Allison, S. G., Moore, T. C., Hare, D. A., Batten, C. F., and Jegley, D. C., in Smart Structures and Materials Conference, SPIE Vol. 4332, 2001, pp. 133-142.

4. M. Lin, 2nd International Workshop on Structural Health Monitoring, ed. by F. K. Chang, Stanford Univ., Technomic, 1999, pp. 603-611.

5. Duke, J. C., Cassino, C. D., Childers, B. A., and Prosser, W. H., "Characterization of an EFPI AE Sensor," submitted to Research in Nondestructive Evaluation (2002).

6. Gorman, M.R., in Review of Progress in QNDE, Vol. 17, eds. D. O. Thompson and D. E. Chimenti, Plenum Press, New York, 1998, pp. 557-564.

7. Prosser, W. H., Hamstad, M. A., Gary, J, and O'Gallagher, A, Journal of Acoustic Emission, Vol. 17(1-2), 1999, pp. 37-47.

8. Woodard, S. E., Coffey, N. C., Taylor, B. D., Brett, R. R. and Woodman, K. L., "Autonomous Health Monitoring Architecture and Hardware," United States Patent Provisional Application and NASA Langley Research Center Invention Disclosure LAR 16407-1, Feb 2002.

9. Castellini, P., and Revel, G. M., "Damage Detection by Laser Vibration Measurement", $15^{\text {th }}$ World Conference on Non-Destructive Testing, Rome, Italy, October 15-21, 2000.

10. Maji A. K., Experimental Mechanics J., Vol. 34, No. 2, 1997, pp. 197-204.

11. Dewherst, R. J., Applied Optics and Optoelectronics IoPP, Bristol, 1996, pp. 289-298

12. Cramer, K. E., and Winfree, W. P., Thermosense XVIII, Orlando, April 8-12, 1996.

13. U. Qidwai, A. H. Costa, and C. H. Chen, Insight, vol. 41, no. 11, 1999, pp.700-703

14. Deobling, S.W.; Farrar, C.R.; Prime, M.B., The Shock and Vibration Digest, Vol. 30 , (1998) 2, pp. 91-105.

15. Krohn, N., Stoessel, R., and Busse, G., in Review of Progress in QNDE, Vol. 20, eds. D. O. Thompson and D. E. Chimenti, AIP, New York, 2000, pp. 1666-1672.

16. Kent, R. M., Murphy, D.A., NASA/CR-2000-209848, January 2000.

17. Munns, T.E., Beard, R.E., Culp, A.M., Murphy, D.A., Kent, R.M., NASA/CR-2000210643, December 2000.

18. Mukkamala, R., Proceedings of the IEEE/AIAA 19th Digital Avionics Systems Conference; 2000 , pp. 6.C.4.1 - 6.C.4.8.

19. Mukkamala, R., Bhoopalam, K., Dammalpati, S., Proceedings of the IEEE/AIAA 20th Digital Avionics Systems Conference; 2001, pp. 3.D.2.1 - 3.D.2.10

20. Munns, T.E., Kent, R.M., Bartolini, A., Gause, C.B., Borinski, J.W., Dietz, J., Elster, J.L., Boyd, C., Vicari, L., Cooper, K., Ray, A., Keller, E., Venkata, V., Sastry, S.C., NASA/CR-2002-211428 February 2002.

21. Doggett, W., Vazquez, S., Proceedings of the IEEE/AIAA 19th Digital Avionics Systems Conference; 2000, pp. 6.D.2.1 - 6.D.2.8 